\title{
LA-UR- $98-2253$
}

\section{STATISTICAL ANALYSIS OF HIGH EXPLOSIVE DETONATION DATA}

Beckman, Fernandez, Ramsay, and Wendelberger

DRAFT $5 / 10 / 98$

\section{INTRODUCTION}

Statistical analysis of data for two different high explosives was performed. The goal of the analysis was to determine how the probability of detonation varies for different run lengths and pressures.

\section{BACKGROUND}

This study investigates the detonation behavior of two different high explosive compounds, PBX 9404 and PBX 9502. One reason these two high explosives were selected is because data is abundant relative to other types of high explosives.

Another reason is that these two explosives represent the high and low ends of the spectrum of HE sensitivity. Sensitivity of an explosive refers to the ease in detonating the explosive. A sensitive explosive is easy to detonate when desired but also carries the risk of inadvertent detonation. An insensitive explosive is more difficult to detonate, but it is safer to use because it is unlikely to detonate when it is not supposed to.

Run length represents an estimate of the length at which a particular high explosive will detonate for a given pressure. If the length of an explosive exceeds the run length, then detonation will occur. Run lengths for PBX9502 and PBX9404 were determined from detonation tests conducted over a range of specified pressures. Since there is random variation in the length at which detonation occurs, run length values may be thought of as an estimate of the mean of the 


\section{DISCLAMER}

This report was prepared as an account of work sponsored by an agency of the United States Government. Neither the United States Government nor any agency thereof, nor any of their employees, makes any warranty, express or implied, or assumes any legal liability or responsibility for the accuracy, completeness, or usefulness of any information, apparatus, product, or process disclosed, or represents that its use would not infringe privately owned rights. Reference herein to any specific commercial product, process, or service by trade name, trademark, manufacturer, or otherwise does not necessarily constitute or imply its endorsement, recommendation, or favoring by the United States Government or any agency thereof. The views and opinions of authors expressed herein do not necessarily state or reflect those of the United States Government or any agency thereof. 


\section{DISCLAIMER}

Portions of this document may be illegible in electronic image products. Images are produced from the best available original document. 
run length distribution.

Table 1 contains run length and pressure data for PBX 9404. Table 2 contains run length and pressure data for PBX 9502. A diagram known as a Pop Plot is commonly used to display the relationship between run length and pressure for high explosives. The Pop Plot plots the Base 10 logarithm of the run length against the Base 10 logarithm of the pressure. Typically, the run-length versus pressure relationship is approximately linear.

Figure 1 shows a pop plot for PBX 9404. Figure 2 shows a pop plot for PBX 9502. Linear regression lines were fitted to each of the two datasets. The fitted lines for PBX 9404 and PBX 9502 are shown in Figure 3. The Pop Plot lines for other types of explosives are expected to lie somewhere between the Pop Plot lines for PBX 9404 and PBX 9502.

\section{PROBLEM DEFINITION}

Several alternative questions about the Pop Plot relationships could be posed. Given a specified run length and pressure, what is the probability of detonation? For a given pressure, what length corresponds to a specified probability of detonation? In this study, we chose to address the following question: For a given length, how does the probability of detonation vary as a function of pressure?

Detonation occurs when the length exceeds the estimated run length. In this study, the run length is not known precisely. Thus, the probability of detonation is taken as the estimate of the probability that the length exceeds the run length. This formulation ignores the variability present in the estimation of run length. However, since the variability about the regression lines is relatively small for the two types of HE studied, this approximation probably does not have much of an effect on the results. 
Once the relationship between probability of detonation and pressure given a specified length is understood, threshhold pressures which lead to specified probabilities of detonation may be determined for a given length. The analysis may be repeated to study multiple lengths of interest.

\section{STATISTICAL APPROACH}

The linear relationships given by the pop plots provide a starting point for examining the probability of detonation. Assuming a bivariate normal distribution for the log transformed pressure and run length data, the linear regression line may be viewed as the conditional mean function of the run length given the pressure. The fitted conditional mean follows a normal distribution, whose parameters can be determined from the parameters of the log run time and $\log$ pressure data. The distribution of the conditional mean may be used to calculate the probability of detonation for a specified length following Anderson (1984).

\subsection{Estimation of Conditional Mean Function}

Let $X_{1}$ be the $\log$ of the run length, $\mathrm{L}$, and let $X_{2}$ be the $\log$ of the pressure, $P$. Suppose that $X_{1}$ and $X_{2}$ are bivariate normal with means $\mu_{1}$ and $\mu_{2}$, variances $\sigma_{1}^{2}$ and $\sigma_{2}^{2}$, and correlation coefficient $\rho$. Then the $\log$ run length given the $\log$ pressure follows a normal distribution with mean $x_{1} \mid \mu_{1}+\left(\sigma_{1} \rho / \sigma_{2}\right)\left(x_{2}-\mu_{2}\right)$ and variance $\sigma_{1}^{2}\left(1-\rho^{2}\right)$. The conditional mean is equivalent to the linear regression of $X_{1}$ on $X_{2}$, i.e., $x^{*} \mid P$ is normally distributed with mean $\alpha+\beta P$ and variance $\sigma_{1}^{2}\left(1-\rho^{2}\right)$ ), where $\alpha$ and $\beta$ are the intercept and slope of the regression of $x^{*}$ on $P$.

\subsection{Calculation of the Probability of Detonation}

Use of the normal distribution allows statistical assessment of the probability that, for a 
given length and specified pressure, the length of the explosive will exceed the run length and will detonate. The assumption of a parametric distribution provides a mechanism for estimating behavior of the variables of interest beyond the range of the original data. However, the reliability of the results will depend on the validity of the original distributional assumptions.

The probability of detonation is estimated as the probability that the length, $L$, exceeds the run length, $x^{*}$, given pressure.

$$
P\left(L>x^{*}\right)=\Phi\left(\left(L-\mu_{x^{*} \mid P}\right) / \sigma_{x * \mid P}^{2}\right),
$$

where $\Phi$ is the cumulative distribution of a standard normal random variable with mean 0 and variance 1 . Note that the conditional variance, $\sigma_{1}^{2}\left(1-\rho^{2}\right)$, does not depend on the pressure.

\subsection{Statistical Bounding of Detonation Probability}

A bootstrap resampling approach was used to examine the variability in the fitted regression line and the resulting calculated probabilities. Efron and Tibshirani (1993) discuss bootstrap resampling methods. The resampling plan used for the analysis of the HE data involved generating 1000 resamples of the original data, estimating the parameters of the bivariate distribution, and using the estimated parameters to compute the probability of detonation for specified pressure values.

The resamples were generated by sampling the original data pairs with replacement. Each resample is the same size as the original data set. Each data pair in a resample is obtained by selecting from the set of all data pairs in the original data set with equal probability. The resamples capture the variability of the data pairs in the original data set. The probability of detonation is calculated for each of the resamples. The set of 1000 values obtained for the probability of detonation provide distributional information on the probability of detonation at 
specified pressures. Selection of a desired percentile of the distribution at several different pressure values provides a bound on the probability of detonation as a function of pressure.

The upper 95th percentiles of the probability values at each pressure value were used to generate a pointwise $95 \%$ upper bound on the probability of detonation as a function of pressure.

\subsection{Determination of a Threshhold Pressure}

A threshhold pressure may be determined such that the probability of detonation is below a specified limit by examining the upper bound on the probability of detonation as a function of pressure. In the figures, a horizontal line is drawn corresponding to a log detonation probability of $10^{-6}$. The point where the horizontal line crosses the dashed upper bound line indicates the pressure at which the log probability reaches $10^{-6}$.

Figures 4-11 illustrate the distributions obtained from the resamples and the upper bounds for the relationship between the log probability of detonation and the log pressure for selected run lengths for PBX 9404 and PBX 9502.

\section{SUMMARY}

In this study, linear regression and resampling methods were used to compute bounds on the probability of detonation for particular lengths as a function of pressure. This methodology can be applied to other lengths to provide further information on sensitivity.

\section{FURTHER QUESTIONS}

Some additional questions remain to be addressed. For the PBX 9502, the data was obtained from two different sources. The first 19 data points were obtained from one set of experiments, while the remaining 25 points were obtained from another set of experiments. Further analysis 
could be conducted to determine whether the points from these different sources are statistically different by examining the change in the amount of variation explained by fitting parameters to one subset of the data relative to the variation explained by fitting parameters to the combined dataset.

Another question that has been raised is how the probability of detonation behaves for other explosives whose sensitivities lie between PBX 9404 and PBX 9502. Presumably, the Pop plots for intermediate sensitivity explosives would lie between the two types investigated here. Since less data is available for other types, the results for PBX 9404 and PBX 9502 might be incorporated into the analysis in some manner to compensate for small sample sizes.

\section{REFERENCES}

1. Anderson, T. W. (1984), An Introduction to Multivariate Statistical Analysis, John Wiley \& Sons, New York, pp. 35-37.

2. Efron, B. and Tibshirani, R. (1993), An Introduction to the Bootstrap, Chapman and Hall, New York. 


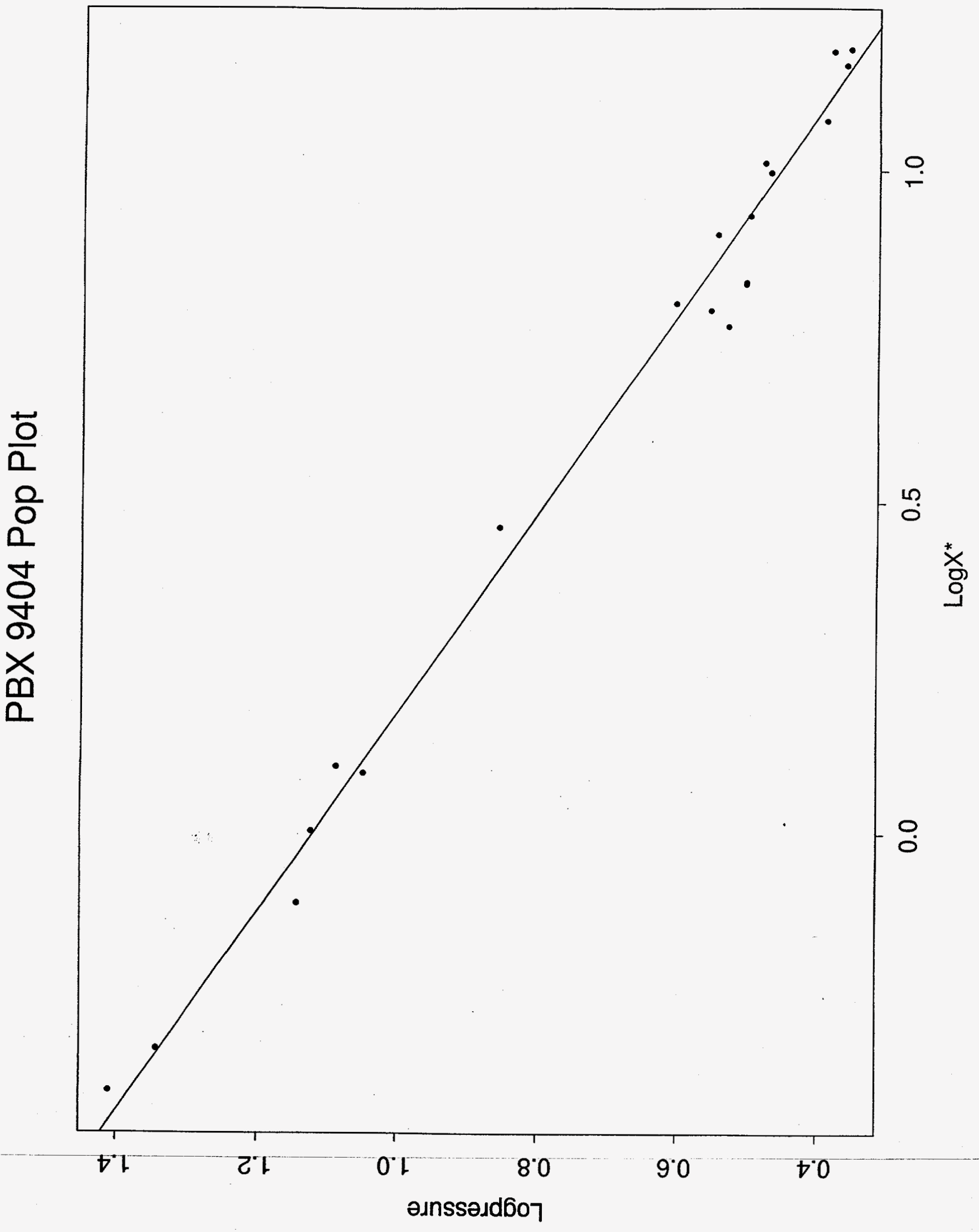




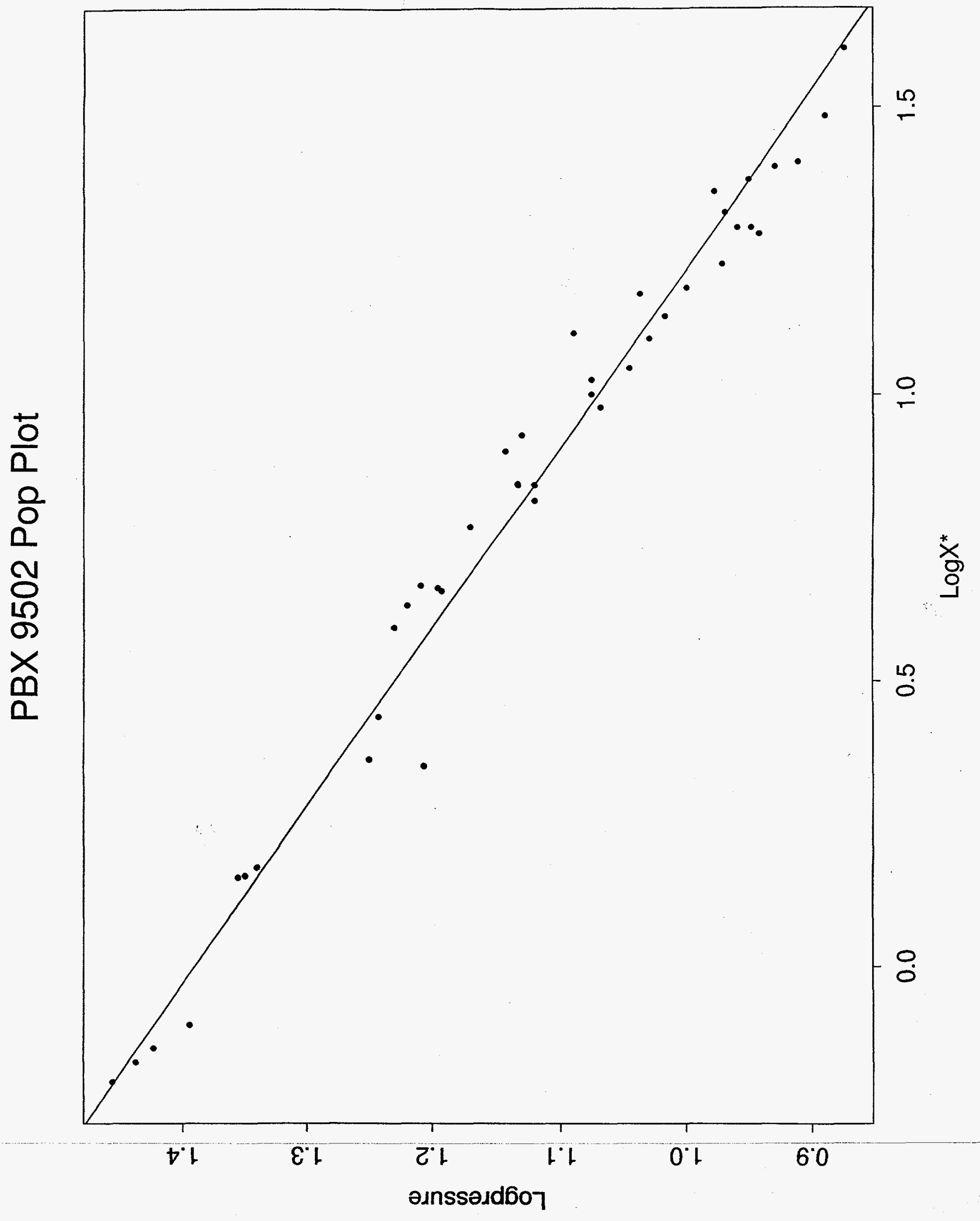




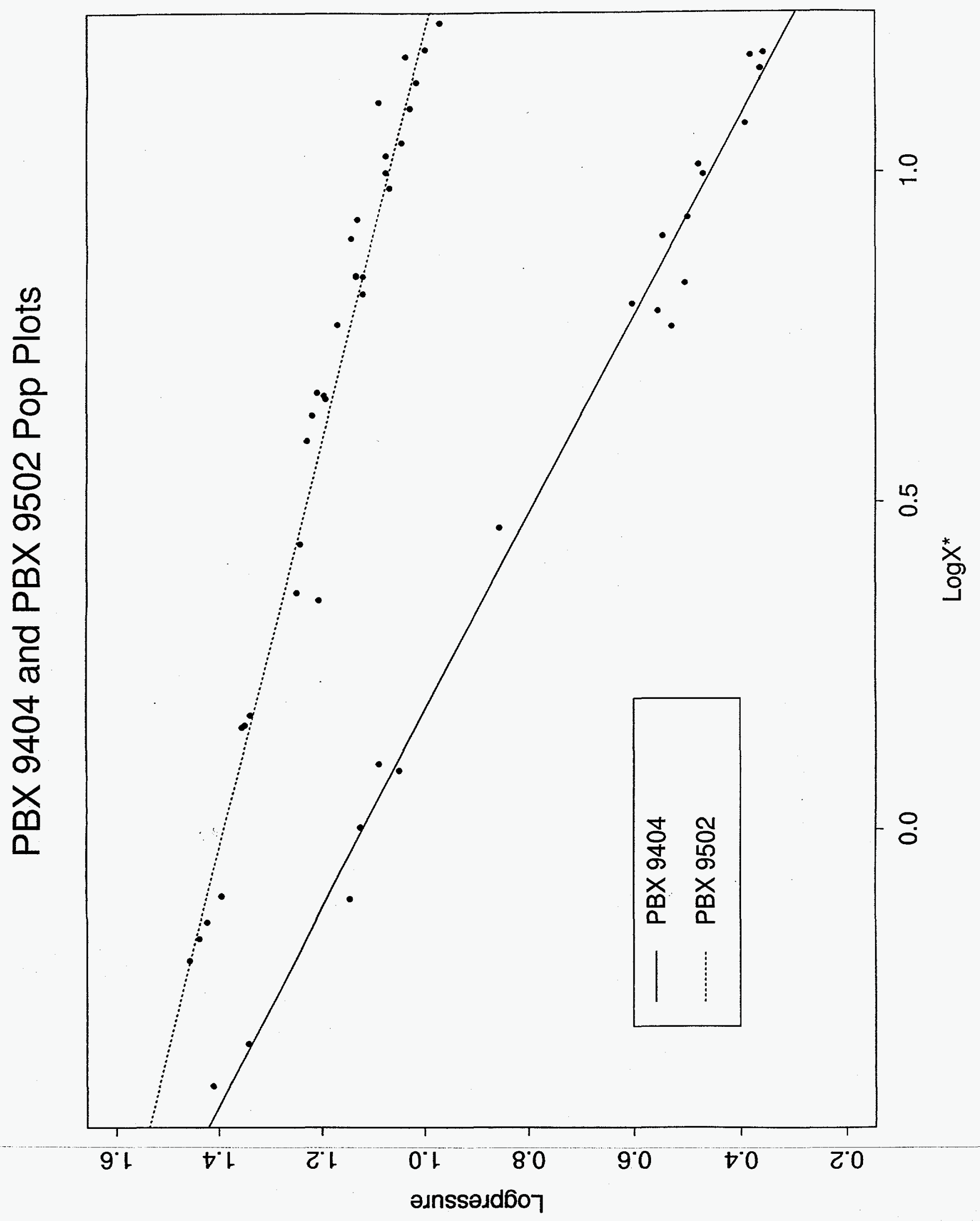




\section{PBX 9502, Run Length $=25$}

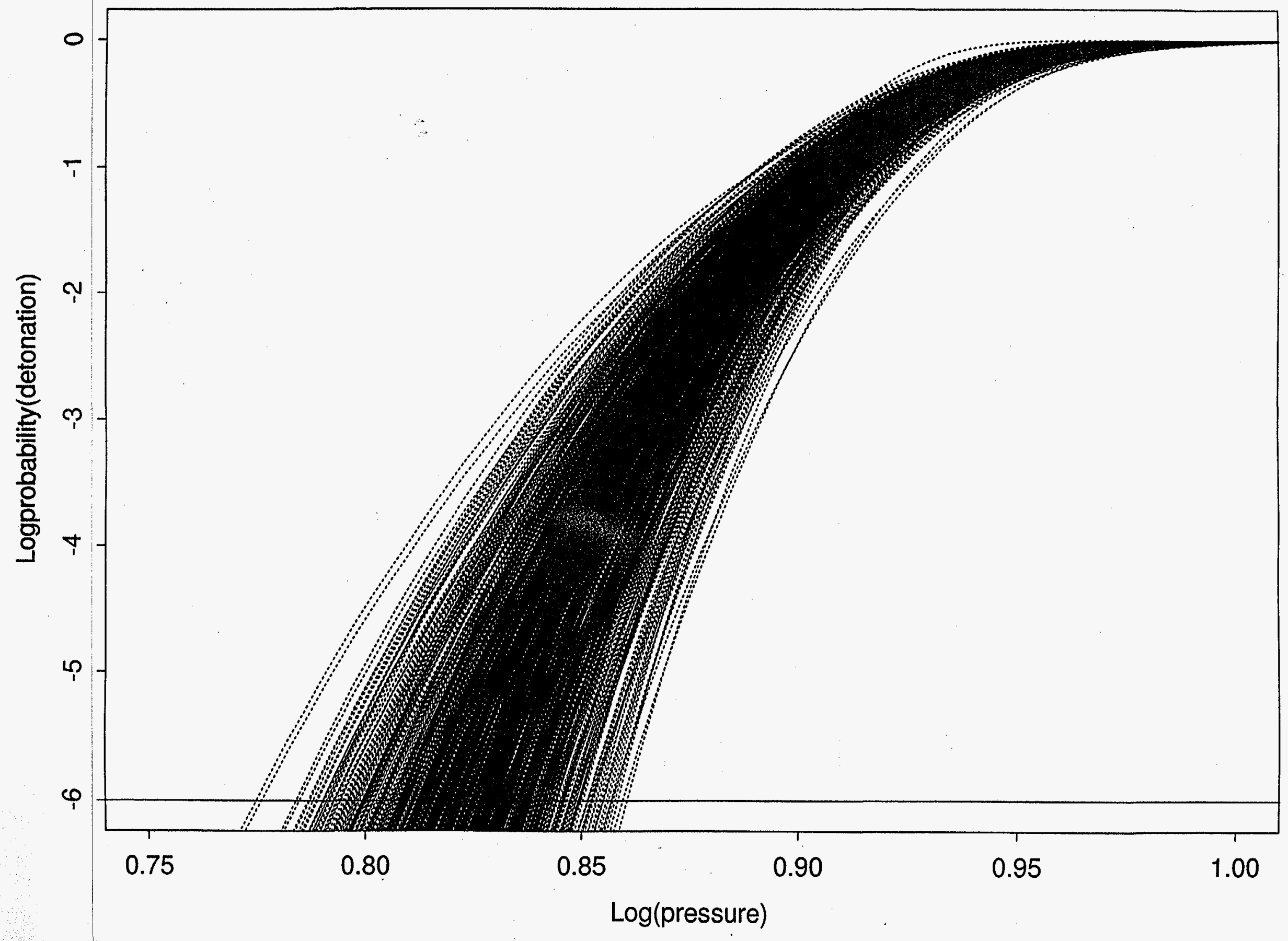




\section{PBX 9502- 95\% Upper Limit, Run Length=25}

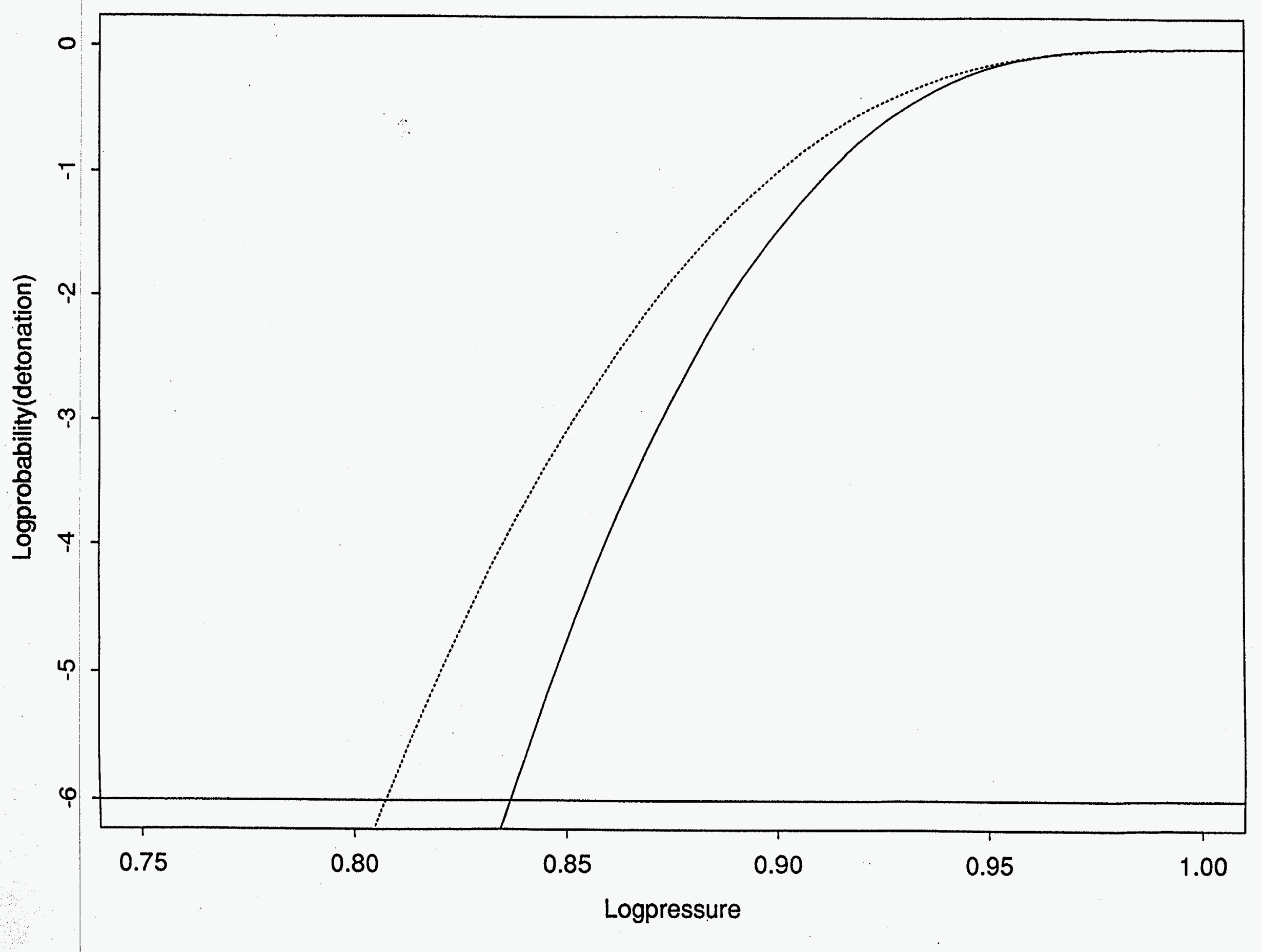




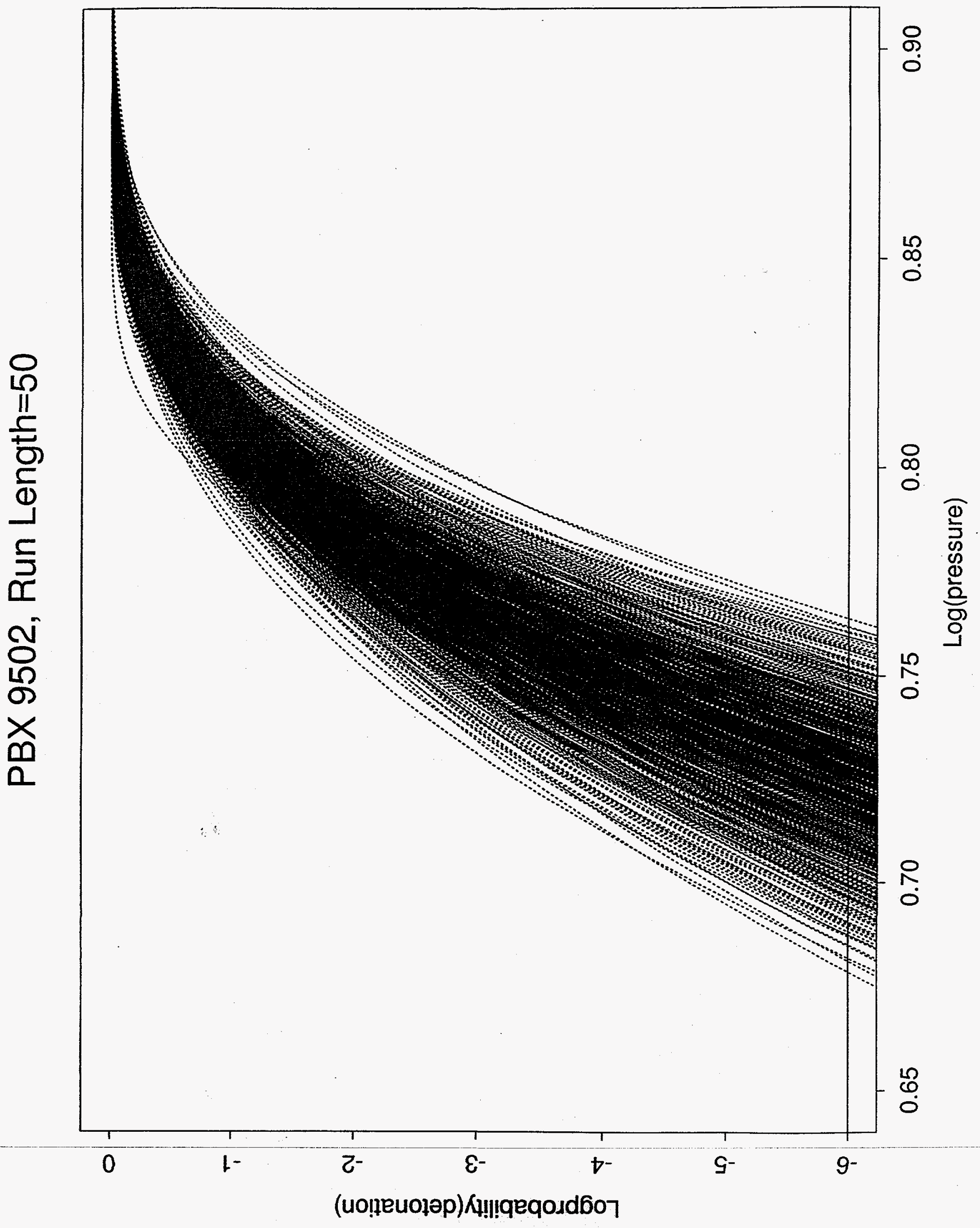




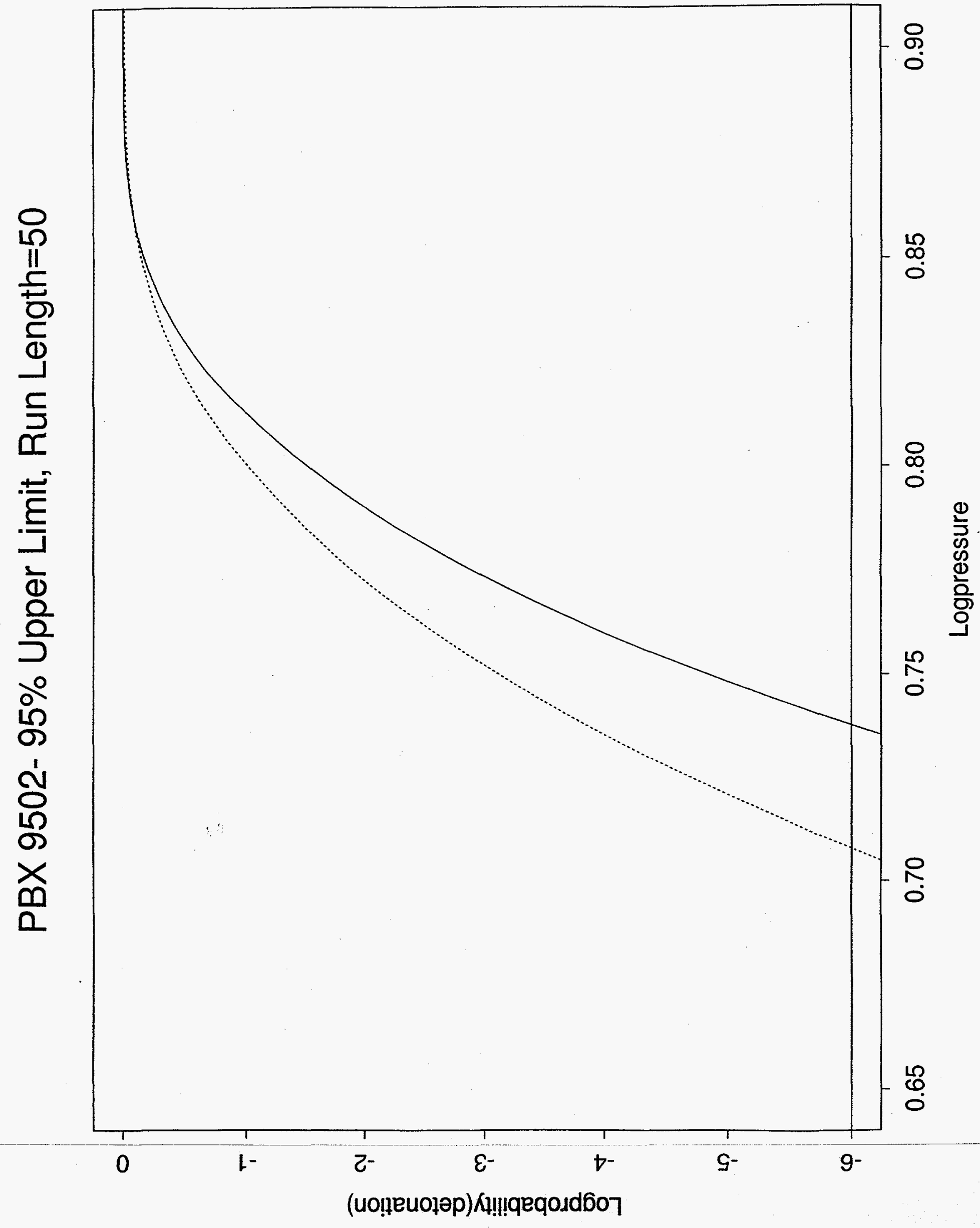




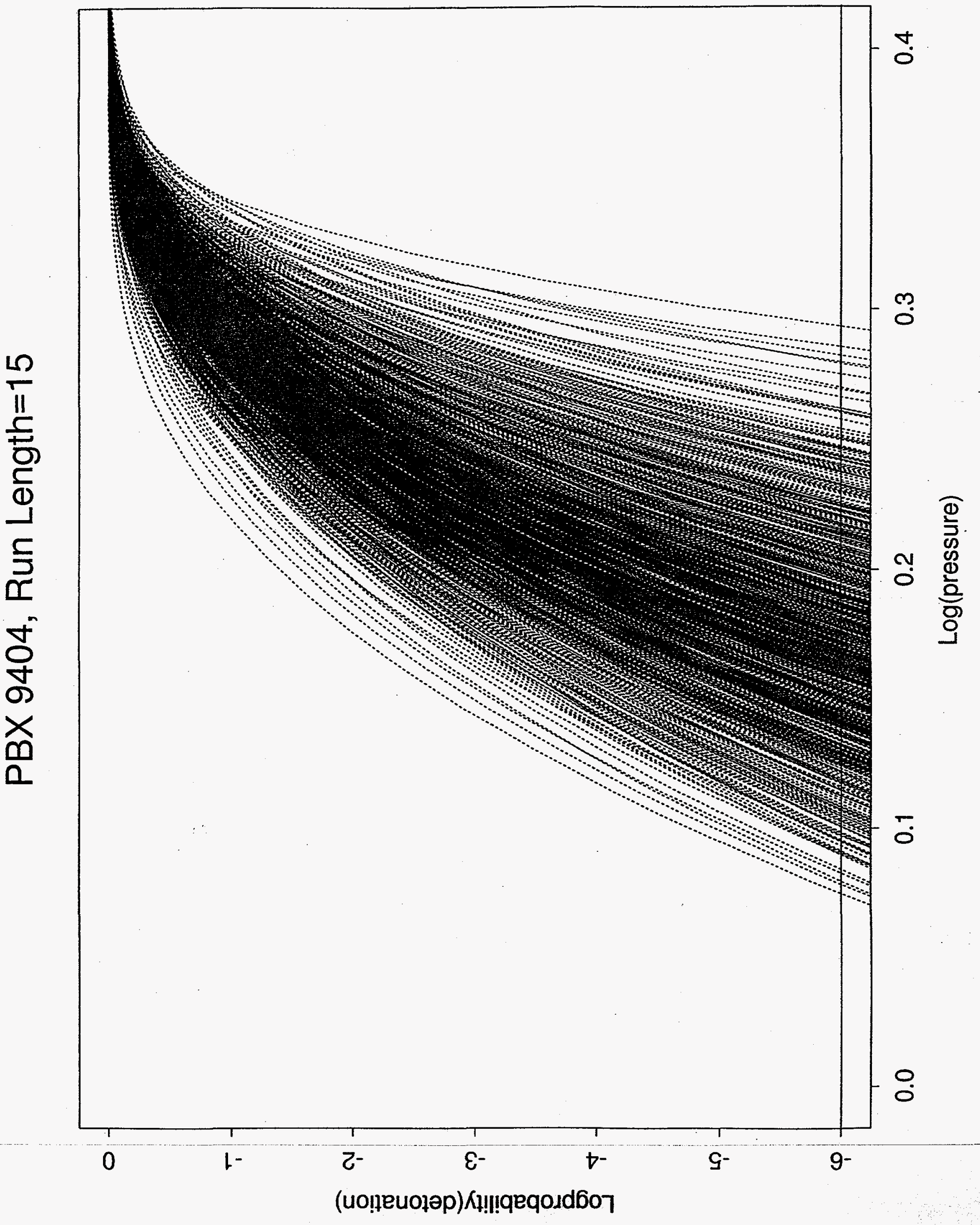




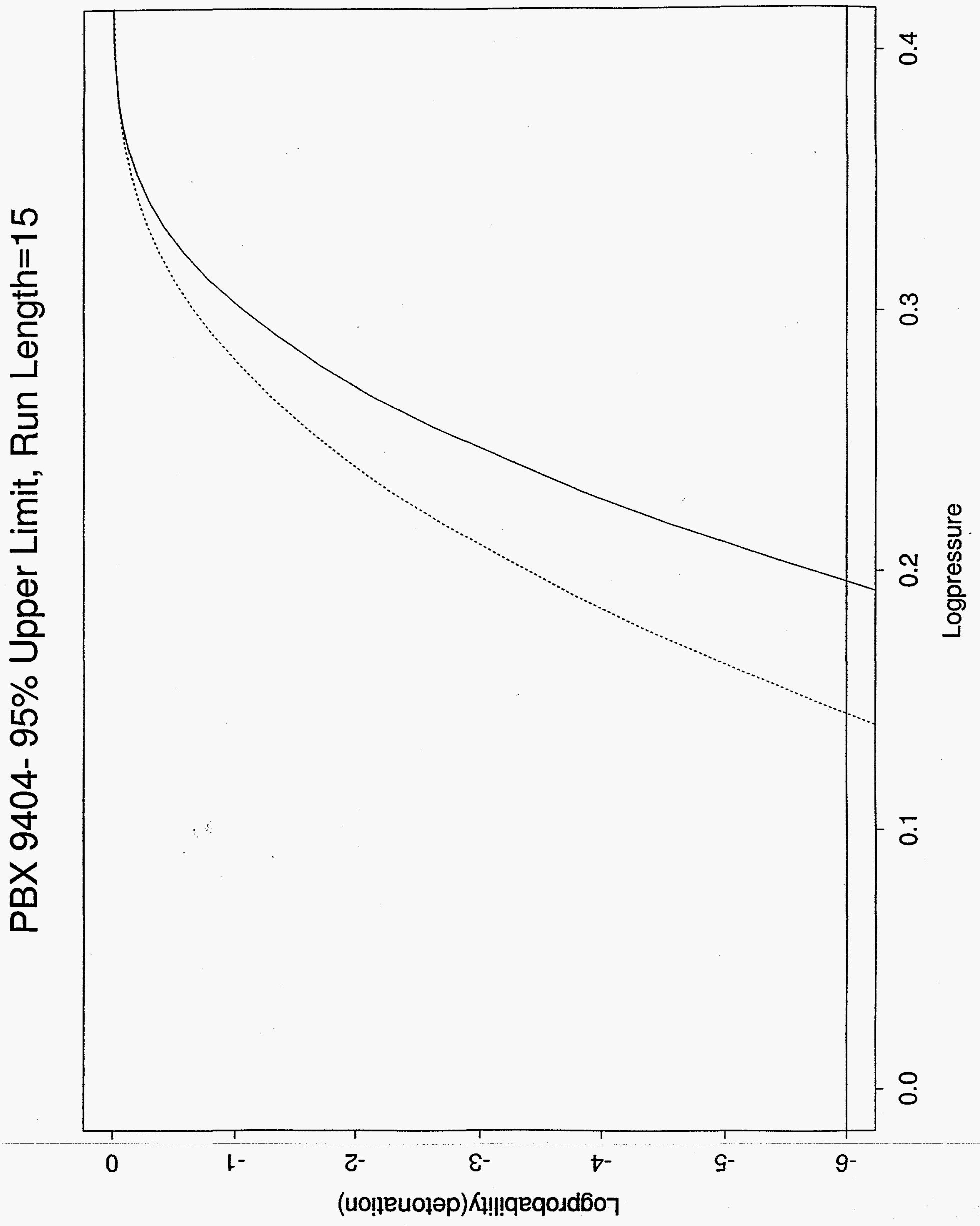




\section{PBX 9404, Run Length $=25$}

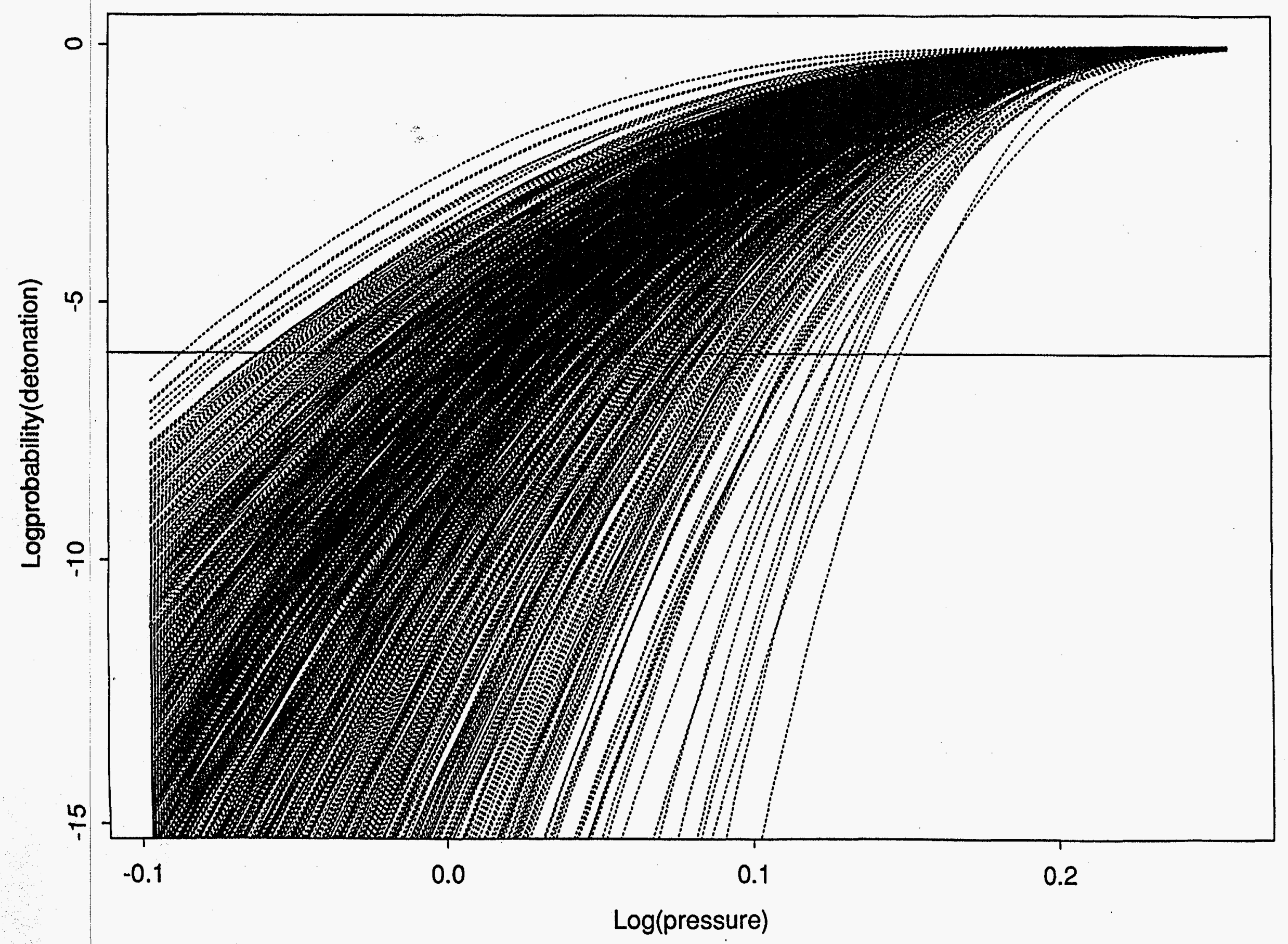




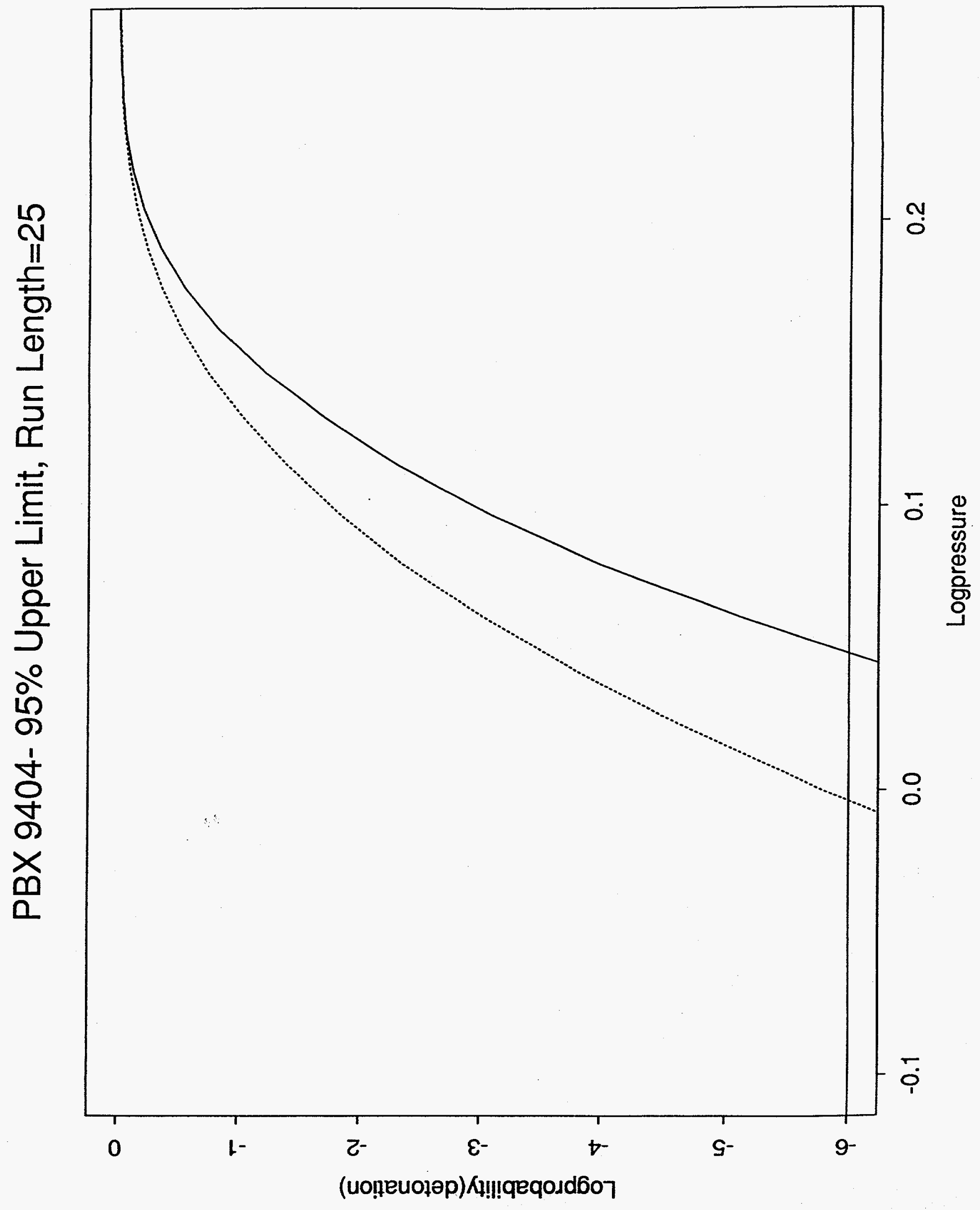

